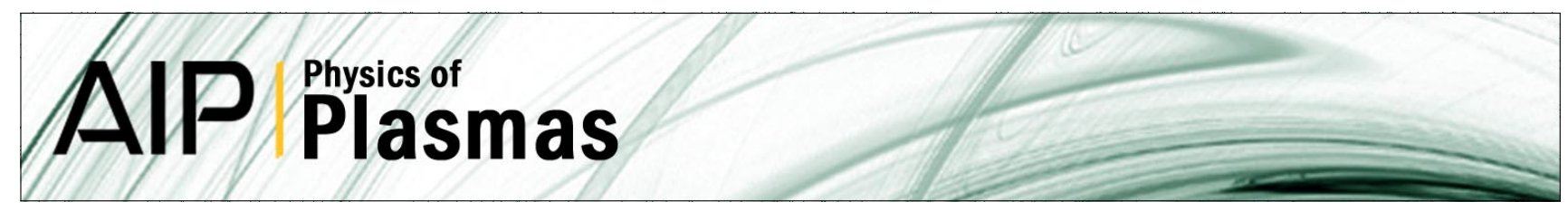

\title{
The beat in laser-accelerated ion beams
}

M. Schnürer, A. A. Andreev, F. Abicht, J. Bränzel, Ch. Koschitzki et al.

Citation: Phys. Plasmas 20, 103102 (2013); doi: 10.1063/1.4824115

View online: http://dx.doi.org/10.1063/1.4824115

View Table of Contents: http://pop.aip.org/resource/1/PHPAEN/v20/i10

Published by the AIP Publishing LLC.

\section{Additional information on Phys. Plasmas}

Journal Homepage: http://pop.aip.org/

Journal Information: http://pop.aip.org/about/about_the_journal

Top downloads: http://pop.aip.org/features/most_downloaded

Information for Authors: http://pop.aip.org/authors

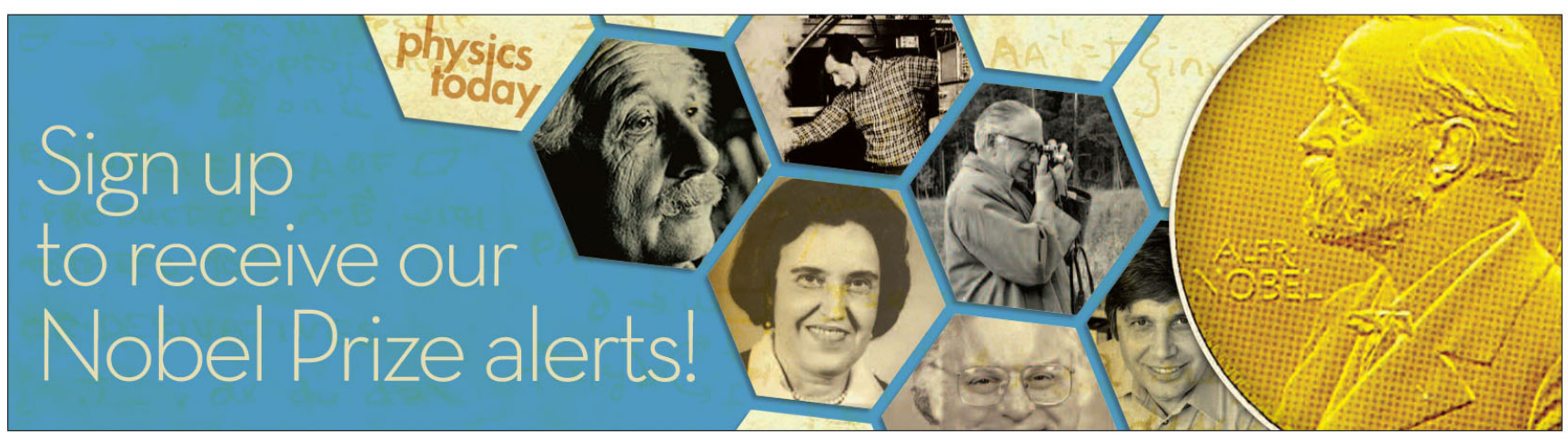




\section{The beat in laser-accelerated ion beams}

M. Schnürer, ${ }^{1, a)}$ A. A. Andreev ${ }^{1,2,3}$ F. Abicht, ${ }^{1}$ J. Bränzel, ${ }^{1}$ Ch. Koschitzki, ${ }^{1}$ K. Yu. Platonov, ${ }^{2}$

G. Priebe, ${ }^{4}$ and W. Sandner $r^{1,5}$

${ }^{1}$ Max-Born-Institut, Max-Born-Str. $2 a, 12489$ Berlin, Germany

${ }^{2}$ Vavilov State Optical Institut, Birzhevaya line 12, 199064 St. Petersburg, Russia

${ }^{3}$ St. Petersburg University, 199064 St. Petersburg, University emb.6, Russia

${ }^{4}$ XFEL GmbH, Notkestr. 85, 22607 Hamburg, Germany

${ }^{5}$ Technical University Berlin, Straße des 17. Juni 135, 10623 Berlin, Germany

(Received 23 July 2013; accepted 19 September 2013; published online 3 October 2013)

Regular modulation in the ion velocity distribution becomes detectable if intense femtosecond laser pulses with very high temporal contrast are used for target normal sheath acceleration of ions. Analytical and numerical analysis of the experimental observation associates the modulation with the half-cycle of the driving laser field period. In processes like ion acceleration, the collective and laserfrequency determined electron dynamics creates strong fields in plasma to accelerate the ions. Even the oscillatory motion of electrons and its influence on the acceleration field can dominate over smoothing effects in plasma if a high temporal contrast of the driving laser pulse is given. Acceleration parameters can be directly concluded out of the experimentally observed modulation period in ion velocity spectra. The appearance of the phenomenon at a temporal contrast of ten orders between the intensity of the pulse peak and the spontaneous amplified emission background as well as remaining intensity wings at picosecond time-scale might trigger further parameter studies with even higher contrast. (C) 2013 AIP Publishing LLC. [http://dx.doi.org/10.1063/1.4824115]

\section{INTRODUCTION}

Laser initiated acceleration of ion bunches results in a very low transversal and longitudinal emittance ${ }^{1}$ being a striking feature of Target Normal Sheath Acceleration (TNSA). ${ }^{2}$ This characteristic is derived from the extremely strong acceleration (at the order of Exa g:g-gravitational acceleration of the earth) during a short period of time (several $100 \mathrm{fs}$ to ps), which starts cold ions attached to a surface of a foil onto well-defined trajectories. The transversal emittance is a prerequisite for ion beam projection techniques being already widely applied in radiographic deflection and imaging investigations. ${ }^{3}$ The very low longitudinal emittance allowed deducing specific source dynamics in TNSA experiments. ${ }^{4}$ Here, we describe a characteristic property of the ion source, which is coupled to the pulsing of the acceleration field itself. This is a direct consequence of the laser field and the linked periodical release of electron bunches, building up the acceleration field in TNSA. Collective and periodic movement of dense electron layers produced by intense laser irradiation of solid surfaces is a well-known phenomenon and the related emission of high harmonic orders of the laser frequency is intensively investigated. ${ }^{5}$ So far it was not assumed that laser driven ion acceleration could exhibit fingerprints of the laser cycle. TNSA is a secondary process where field averaging over a longer timescale seems to dominate and because of their inertia the response of the ions in the field is orders of magnitude slower as compared to the electrons. The presented results show that faint modulations in the ion velocity distribution become detectable with a

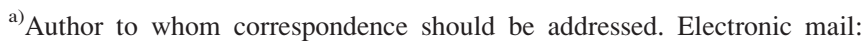
schnuerer@mbi-berlin.de
}

spectrometer of reasonable resolution. Furthermore, the analysis and comparison to previous experiments ${ }^{6}$ suggest an evident role of the temporal contrast of the laser pulse. As well created magnetic fields in the plasma can render the proton spectrum as discussed by Robinson et $a l^{7}$ and can produce a few distinct peaks in the spectrum. Our observation includes a qualitatively and quantitatively different modulation with relatively high frequency.

\section{EXPERIMENT AND SETUP}

Proton beams have been produced by laser irradiated Ti-foils ( 5 micron thick) and its adherent $\mathrm{CH}$-contamination at an intensity of $\sim 3 \times 10^{19} \mathrm{~W} / \mathrm{cm}^{2}$. The used Ti:Sapphire-laser (maxed spec 70 TW-35 fs) is a part of the "High Field Laser" system at Max-Born-Institute Berlin. The XPW-frontend ${ }^{8}$ serves for an amplified spontaneous emission (ASE) background level of $10^{-10}$. Resulting focal intensities are based on calculation with pulse duration, with pulse energies measured in front of the grating compressor, measured relative transmission of grating compressor and beam-line inclusive focusing optics as well as the measured focal spot-sizes with encircled energy content. The irradiation geometry is set with a p-polarized laser field and a $10^{\circ}$ angle of incidence.

Protons (and ions) are registered with a modified Thomson spectrometer. The geometry of the setup is depicted in Fig. 1. The entrance slit of the spectrometer has a variable width of several 100 microns and a length of about $8 \mathrm{~mm}$ (orientation along $\mathrm{z}$-axis). The projection of the source (perpendicular to the slit length-y-axis) to the detector is about 1:1. Propagation of ions is along $\mathrm{X}$-axis, the dispersion of the ions due to the B-field of the spectrometer is along $y$-axis. The resolution of the recorded kinetic energies concerning the used slit width, magnetic field strength, and drift 


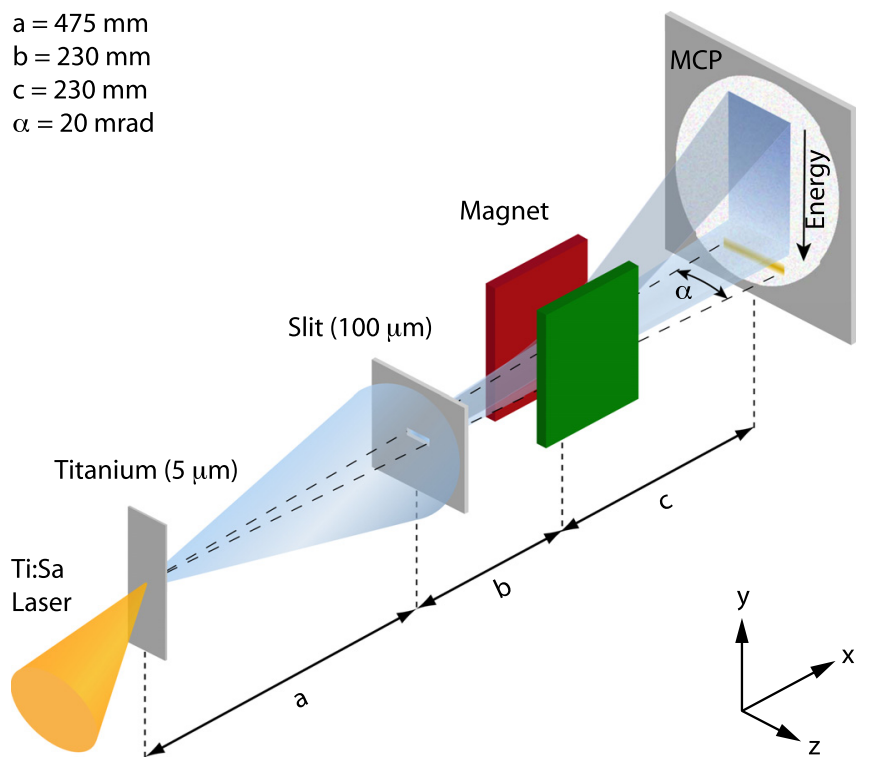

FIG. 1. Scheme of the experiment and convention of coordinate axis used in text description.

length is about (2-5)\% of calculated kinetic energy values. The detector is an imaging MCP (Hamamatsu Type F2226$24 \mathrm{PF} 159$ with $75 \mathrm{~mm}$ diameter active area) coupled to a phosphorous screen, which is photographed by a CCDcamera. Single and multiple particle response calibration ${ }^{9}$ of the detector system has been done with a ${ }^{241} \mathrm{Am}$-source. The imaging quality of the detector is verified with laser accelerated proton beams and projection imaging of test objects.

\section{EXPERIMENTAL RESULTS}

Fig. 2 shows recorded pictures concerning the kinetic energy distribution of a produced ion beam. The electric field for separation of ion species in the spectrometer is switched off. From previous investigation, ${ }^{10}$ we know that with femtosecond pulse drive and high temporal contrast at optimum focus position, a predominant proton signal with a lower carbon ion back ground is given. This holds for the TNSA-

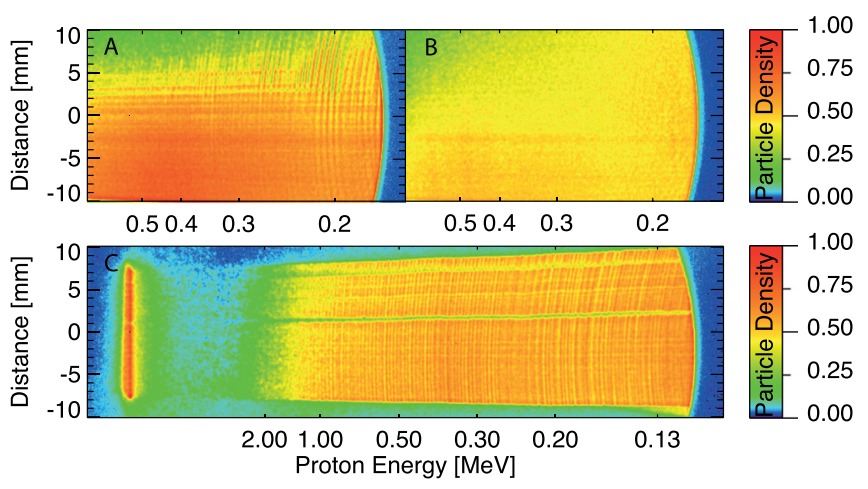

FIG. 2. Photos of a recorded proton (ion) spectra obtained with a MCP-detector: a 5 micron thick Ti-foil plus contamination was irradiated with a $3 \times 10^{19} \mathrm{~W} / \mathrm{cm}^{2}$ ( $\sim 40 \mathrm{fs}$ ) p-polarized laser pulse having a ASE to peak intensity contrast of $10^{-10}$, entrance slit of spectrometer has a width of 100 micron and a length of $8 \mathrm{~mm}$, modulations are clearly detectable in this configuration in (A) and (C) with slightly different spectrometer dispersion, but disappear in (B) — an adjacent shot to (A) — when a thin scatter foil is placed in the beam (cf. text). regime of micrometer thick metal foils as applied in this experiment. Therefore, we attribute the faint but clearly visible modulation of short period to the proton signal. These more than hundred lines are visible with a slit-width of 100 micrometer and a corresponding high energy resolution. The modulation got blurred if the slit-width was increased up to 5 times but features with stronger modulation depth are still visible in some part of the spectrum. In order to exclude that the observation is due to an artifact of detection or of the spectrometer, also spectrograms (Fig. 2(b)) have been recorded with the same setup and laser-shot parameter but placing a 0.8 micrometer thick aluminum foil in the propagation path of the ion beam. (This beam filter was located $2 \mathrm{~cm}$ downstream from the ion source.) Small scale scattering in the filter is already sufficient to wash out the modulation. Hence the observed modulation is an intrinsic beam property. Using a similar detection setup ${ }^{6}$ together with a laser having a peak to ASE-intensity level of about $10^{7}$ has not led to similar observations. Measurement of the temporal pulse shape $^{8}$ applied in this experiment shows intensity wings starting to decrease the $10^{10}$ peak to ASE contrast ratio at about 30 ps in front of the pulse peak. At present, we cannot speculate whether or not the observed phenomenon is coupled only to a contrast of specific high order or also to higher values (e.g., realized with a plasma mirror).

Read out of an integrated ion signal along the slit-length (1D_-spatial co-ordinate $z$ ) as a function of kinetic energy needs some processing of the Fig. 2 picture. The visible bending of all the lines in the spectrum is due to the weak variation of the magnetic field in the U-type magnet. (A complex field array has been taken with a scanning measurement setup.) Also the appearance of such a characteristic curvature gives an additional proof of an inherent energy dependent beam property. Concerning Fig. 2(c), the average field is about $0.28 \mathrm{~T}$ along $5 \mathrm{~cm}$. The $1 \mathrm{~cm}$ gap between the field plates sets the limit of the 1D-spatially resolved observation angle to 15 mrad. Accounting for the B-field variation, integration and signal counting concerning the relevant resolution provides the kinetic energy distribution of protons presented in Fig. 3.

Additionally, data readout and processing between 0.4 MeV and $1.3 \mathrm{MeV}$ is shown in the insert of Fig. 3 in which background (the average-non-fluctuating signal) subtraction is applied to detect maxima and minima positions. The appearance of the modulation is not a strong effect and

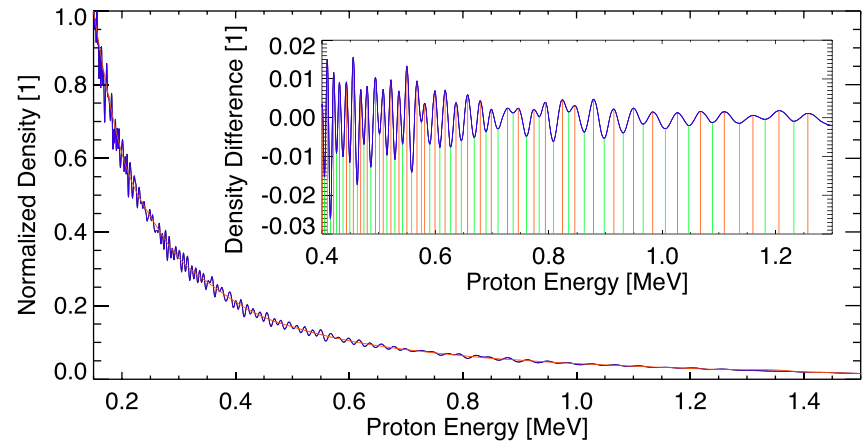

FIG. 3. Integrated (along spatial coordinate z) line-out and determined proton spectrum from Fig. 2(c), insert: cutout and background (main signalred line) subtraction to visualize the modulation feature. 
it is clearly imaginable that from a single spectral trace without spatial information, it is difficult to discriminate between noise and a weak modulation structure with about hundred lines. This reflects the situation if entrance pinholes of a few hundreds of micrometers are used for Thomson Mass Spectrometer setups applied in laser driven ion acceleration. In our case, the 2D picture (1D-spatial resolution versus energy resolution) shows immediately the appearance of a modulation structure.

\section{NUMERICAL SIMULATION AND ANALYTICAL MODEL DISCUSSION}

For interpretation and understanding of our findings, we start with numerical simulation and develop out of it an analytical model. Particle in cell (PIC)-simulation has been done with a modified $2 \mathrm{D}$ LSP-code ${ }^{11}$ using 30 particles in a cell of $4 \mathrm{~nm}$ times $12 \mathrm{~nm}$ and calculating a simulation box of 50 microns times 50 microns. The laser irradiation geometry is identical to the experiment using a slightly higher laser intensity of $5 \times 10^{19} \mathrm{~W} / \mathrm{cm}^{2}$ being incident on a 1 micron thick Ti-foil with a $50 \mathrm{~nm}$ H-layer attached to the rear side of the foil. The densities are solid density and the initial density profile has a rectangular shape. The temporal and spatial distribution of the laser field use super-Gaussian (order of 8) functions with 10 oscillation periods $\mathrm{T}_{\mathrm{L}}$ (for $\lambda_{\mathrm{L}} \approx 0.8 \mu \mathrm{m}$ : $\mathrm{T}_{\mathrm{L}} \approx 2.7 \mathrm{fs}$ ) and a spot size of 4 microns, respectively. Temporal pulse and target density distribution approximate the starting conditions for interaction with very high temporal intensity contrast.

The simulation clearly shows creation of distinct electron bunches having a temporal separation of $T_{L} / 2$. The situation is not unexpected because it reflects the cyclic action of the ponderomotive force in our irradiation geometry. Resulting temporal modulation in the electrical sheath field are visible if the data have sub-cycle resolution. This temporal modulation translates into a modulation of the calculated proton distribution function. In Fig. 4(a), the kinetic energy distribution of protons is shown at a time moment of $200 \mathrm{fs}$ after the laser-target interaction has started. In order to test that the modulation is not due to numerical noise, different runs with different resolution have been performed which validated the simulation result. Similar to the experimental result, the modulation period (in terms of energy) is larger and smaller for larger and smaller proton energies, respectively. We can compare the number of maxima/minima in certain energy intervals from measurement and simulation. The measurement out of the experimentally observed spectra shows the final result of acceleration. The distribution (time instant 200 fs) in Fig. 4(a) will develop further in time. As it is very costly to calculate the proton distribution function with high accuracy in our conditions at a time of already 200 fs, thus we calculated with bigger steps. In this case, the distribution was shifted to higher energies but keeping the same characteristic. Therefore, one can expect that a calculation with high accuracy would give us a small shift in proton energies but with quite similar modulation. If one considers that the calculated time duration $=200 \mathrm{fs}$ is close to the final acceleration instant, we can compare the number of maxima visible in numerical simulation (Fig. 4(a)) and experiment

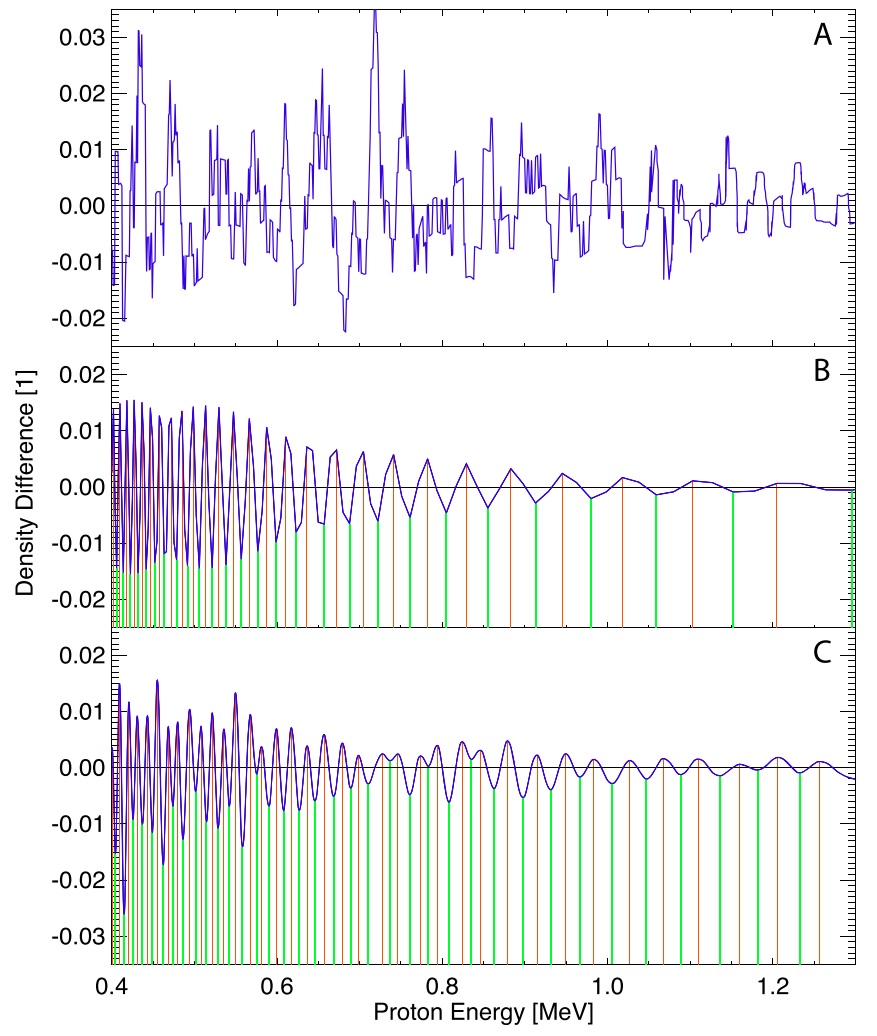

FIG. 4. A-Calculated proton distribution function from 2D PIC simulation (parameter cf. text) at a time moment $200 \mathrm{fs}$ after laser-target interaction has started, B-analytical 1D model calculation, and C-experimental result.

(Fig. 4(c)). In the energy interval $[0.8,1.2] \mathrm{MeV}$, the number of maxima/minima is quite similar; where at lower energies, probably the resolution of the numerical simulation cannot account to resolve the modulation frequency obtained in experiment.

The modulation of electron density in space and time and its direct relation to oscillations of the ponderomotive pressure is an essential finding from simulation and it will be used now in the form of a perturbation ansatz for an analytical 1D model. Fast electron bunch propagation, modulation, and energy spread are expressed via

$$
\begin{aligned}
\mathrm{n}_{\mathrm{e}} & =\mathrm{n}_{\mathrm{e} 0} \mathrm{e}^{\frac{\mathrm{e} \varphi}{T_{e}}}\left(1+\frac{\mathrm{n}_{1 \mathrm{e}} \mathrm{e}^{-\mathrm{t}^{2} / \mathrm{t}_{\mathrm{L}}^{2}}}{\mathrm{n}_{\mathrm{e} 0}} \cos ^{2}\left(\mathrm{k}\left(\mathrm{x}-\mathrm{tv}_{\mathrm{e}}{ }^{(0)}\right)\right)\right), \\
\mathrm{v}_{\mathrm{e}}{ }^{(0)} / \mathrm{c} & =\sqrt{1-\frac{1}{\left(1+\left(\varepsilon_{\mathrm{e} 0}+\mathrm{e} \varphi\right) / \mathrm{m}_{\mathrm{e}} \mathrm{c}^{2}\right)^{2}}}
\end{aligned}
$$

with $\mathrm{n}_{\mathrm{e}}$ is electron density, $\mathrm{n}_{\mathrm{e} 0}$ is initial electron density, $\mathrm{n}_{\mathrm{e} 1}$ is electron density perturbation, $\mathrm{T}_{\mathrm{e}}$ is electron temperature, $\mathrm{e}-\mathrm{m}_{\mathrm{e}}$ are electron charge and mass, $\varphi$ is electric potential, $\mathrm{c}$ is velocity of light, $\varepsilon_{\mathrm{e} 0}$ is initial energy of electron, $\mathrm{k}-, \mathrm{t}_{\mathrm{L}}$ are wave vector and laser pulse duration, and $(\mathrm{x}, \mathrm{t})$ is propagation-coordinate and time.

The ion motion is described with hydro and Poisson equations similar to the work of Mora; ${ }^{11}$ and for their solution, a perturbation method is used taking into account $\delta \mathrm{n}_{1 \mathrm{e}}=\mathrm{n}_{1 \mathrm{e}} / \mathrm{n}_{\mathrm{e} 0} \ll 1$. In the following analysis, we are using 
only the particular solution of an inhomogeneous system of differential equations. Our relevant initial and boundary conditions favor to omit the eigenmodes of plasma oscillations as it is confirmed by numerical analysis. Therefore, we can also neglect the solution of the homogeneous system. In zero approximation $\left(\delta \mathrm{n}_{1 \mathrm{e}}=0\right)$, we choose the solutions for the ion (proton) kinematics as well from Mora ${ }^{11}$

$$
\begin{aligned}
& \mathrm{v}_{\mathrm{i}}{ }^{(0)}=\mathrm{x} / \mathrm{t}+\mathrm{c}_{\mathrm{s} 0} \quad \mathrm{n}_{\mathrm{i}}^{(0)}=\mathrm{n}_{\mathrm{i} 0} \exp \left(-\mathrm{x} / \mathrm{c}_{\mathrm{s} 0} \mathrm{t}\right), \\
& \mathrm{e} \varphi^{(0)}=\mathrm{T}_{\mathrm{e}} \ln \left(\mathrm{Zn}_{\mathrm{i}}{ }^{(0)} / \mathrm{n}_{\mathrm{e} 0}\right), \quad \mathrm{c}_{\mathrm{s} 0}=\left(\mathrm{ZT}_{\mathrm{e}} / \mathrm{m}_{\mathrm{i}}\right)^{1 / 2}, \quad \mathrm{Zn}_{\mathrm{i} 0}=\mathrm{n}_{\mathrm{e} 0} \\
& \mathrm{f}^{(0)}(\varepsilon)=\frac{\mathrm{dn}_{\mathrm{i}}{ }^{(0)}}{\mathrm{m}_{\mathrm{i}} \mathrm{v}_{\mathrm{i}}{ }^{(0)} \mathrm{dv}_{\mathrm{i}}{ }^{(0)}}=\frac{\mathrm{n}_{\mathrm{i} 0} \exp \left(1-\sqrt{2 \varepsilon / \mathrm{T}_{\mathrm{e}}}\right)}{\sqrt{2 \varepsilon \mathrm{T}_{\mathrm{e}}}},
\end{aligned}
$$

with $n_{i}$ is fast ion density, $v_{i}$ is ion velocity, $Z$ is ion charge, $\mathrm{m}_{\mathrm{i}}$ is ion mass, $n_{i 0}$ is initial proton density, and $\mathrm{f}^{(0)}(\varepsilon)$ is proton distribution function on energy $\varepsilon$ in zero approximation. In linear (first) approximation, density, velocity, and potential function are developed with additional perturbation terms accounting for fast—connected to the laser cycle — changes in time and space

$$
\begin{aligned}
& \mathrm{n}_{\mathrm{i}}^{(1)}=\mathrm{n}_{10} \cos \left(2 \mathrm{k}\left(\mathrm{x}-\mathrm{v}_{\mathrm{e}}{ }^{(0)} \mathrm{t}\right)\right), \\
& \mathrm{v}_{\mathrm{i}}{ }^{(1)}=\mathrm{v}_{10} \cos \left(2 \mathrm{k}\left(\mathrm{x}-\mathrm{v}_{\mathrm{e}}{ }^{(0)} \mathrm{t}\right)\right), \\
& \varphi^{(1)}=\varphi_{10} \cos \left(2 \mathrm{k}\left(\mathrm{x}-\mathrm{v}_{\mathrm{e}}^{(0)} \mathrm{t}\right)\right) .
\end{aligned}
$$

Inserting $(2,3)$ in the hydro and Poisson equations, one can get in first approximation a linear system of algebraic equations for the unknown amplitudes $n_{10}, v_{10}, \varphi_{10}$ taking into account a weak temporal and spatial dependence of the variables in (2) in comparison to those in (3). Solving this system, we obtain

$$
\begin{aligned}
\varphi_{10} & \left.=-\frac{\pi e n_{1 e} e^{-t^{2} / t_{L}{ }^{2}} n_{i}{ }^{(0)}}{2 k^{2} n_{e 0}}, v_{10}=-\frac{Z \pi e^{2} n_{1 e} e^{-t^{2} / t_{L}{ }^{2}} n_{i}{ }^{(0)}}{2 k^{2} n_{e 0} m_{i}\left(v_{e}(0)-v_{i}(0)\right.}\right) \\
n_{10} & =-\frac{Z \pi e^{2} n_{1 e} e^{-t^{2} / t_{L}{ }^{2}} n_{i}{ }^{(0)^{2}}}{2 k^{2} n_{e 0} m_{i}\left(v_{e}{ }^{(0)}-v_{i}{ }^{(0)}\right)^{2}} .
\end{aligned}
$$

Finally, an expression for the proton distribution function is obtained as $f=d n_{i} / m_{i} v_{i} d v_{i}$ and determined by the following parametric dependence:

$$
\begin{aligned}
& f(\tau)=\frac{n_{i 0}}{Z T_{e}}\left(\begin{array}{l}
\frac{\exp (-\xi / \tau)}{1+\xi / \tau}\left(1+\frac{2 \beta_{e}{ }^{(0)} \beta_{10} \sin \left(2\left(\xi-\tau \beta_{e}{ }^{(0)}\right)\right)}{\xi / \tau^{2}}\right) \\
+\frac{2 \beta_{e}{ }^{(0)} N_{10} \sin \left(2\left(\xi-\tau \beta_{e}{ }^{(0)}\right)\right)}{(1+\xi / \tau) \xi / \tau^{2}}-\frac{\beta_{10} \exp (-\xi / \tau) \cos \left(2\left(\xi-\tau \beta_{e}{ }^{(0)}\right)\right.}{(1+\xi / \tau)^{2}}
\end{array}\right) \\
& \varepsilon(\tau)=Z T_{e}\left(1+\xi / \tau+\beta_{10} \cos \left(2\left(\xi-\tau \beta_{e}{ }^{(0)}\right)\right)\right)^{2} / 2, \quad \tau \in\left[\xi T_{e} / \varepsilon_{e 0} ; \infty[,\right.
\end{aligned}
$$

where $\xi=k x, \tau=k c_{s} t, \beta_{e}^{(0)}=v_{e}^{(0)} / c_{s}, \beta_{10}=v_{10} / c_{s}, N_{10}$ $=n_{10} / n_{i 0}$. From simulation, the values $\varepsilon_{e 0}, T_{e}, \delta n_{1 e}$ are 14 $m_{e} c^{2}, 0.6 m_{e} c^{2}$, and 0.02 , respectively. The derivative of the distribution function $f^{(1)}=f-f^{(0)}$ at $\xi \gg 1$ (dependence on $\xi$ is negligible) is depicted in Fig. 4(b). The trend of an increasing modulation period with higher proton energies is clearly reproduced and the number of maxima/minima in certain energy ranges is similar in model and experiment. Here, the number corresponds well for lower energies (cf. to $[0.4,0.6] \mathrm{MeV})$. Due to the applied low order approximation in the model, one can only reproduce the number of peaks in a limited but adjustable spectral range. Changes of the modulation period are connected to electron bunch deceleration in an ambipolar field. The theoretical analysis gives strong indication that the observed modulation in the distribution function of proton energies is related to the half-period of the laser cycle $T_{L} / 2$. Thus, we can use these relative time stamps reversely for conclusions concerning the acceleration process. The experimental result shows indeed that TNSA ion beams (or ion bunches) are well ordered in time (similar to a chirped optical pulse) and the longitudinal emittance is extremely low. Assuming an insignificant change of acceleration during $\sim 1$ fs, we can easily estimate the acceleration $a_{p}$ of protons with mass $m_{p}$ during the time when adjacent energy maxima $E_{p, I}$ and $E_{p, i+1}$ are created: $\left(2 E_{p, I} / m_{p}\right)^{1 / 2}$ - $\left(2 \mathrm{E}_{\mathrm{p}, \mathrm{I}+1} / \mathrm{m}_{\mathrm{p}}\right)^{1 / 2} \approx \mathrm{a}_{\mathrm{p}} \mathrm{T}_{\mathrm{L}} / 2$. The acceleration $\mathrm{a}_{\mathrm{p}}$ is in the order of $10^{20} \mathrm{~m} / \mathrm{s}^{2}$, which can be also expressed with an accelerating electrical field of about $1 \mathrm{MV} / \mathrm{micron}$. These numbers had been also deduced from several previous model calculations and simulations. Now we can directly derive them from a distinct experimental observation and one basic assumption. From our results, we obtain lower acceleration and corresponding lower field strength for lower values of proton energies. Using our modulation as definite time stamps, we can conclude how the acceleration decreases in time, which is possible to fit with a function including the time at a power of 2. Also this corresponds well to accepted model considerations. ${ }^{12}$

\section{CONCLUSION}

Summarizing we found weak modulation in the distribution function of laser generated proton beams (bunches) if the TNSA-scheme has been applied and femtosecond laser pulses with very high temporal contrast have been used. Comparison with theoretical analysis including simulation and analytical model calculation gives strong indication that the modulation is associated with the pulsing laser field and 
that the period of modulation is connected with the halfcycle of the driving laser field. Using this as the only premise, one can deduce acceleration parameters from the experimental observation. Probably, more refined analysis with the whole data set of the observed phenomenon is possible.

\section{ACKNOWLEDGMENTS}

The research leading to these results has received funding from Deutsche Forschungsgemeinschaft within the program CRC/Transregio 18 and from LASERLAB-EUROPE (Grant Agreement No. 284464, EC's Seventh Framework Program). A.A.A. acknowledges the provided computation resources of JSC at project HBUIS.

${ }^{1}$ T. E. Cowan, J. Fuchs, H. Ruhl, A. Kemp, P. Audebert, M. Roth, R. Stephens, I. Barton, A. Blazevic, E. Brambrink, J. Cobble, J. Fernández, J. C. Gauthier, M. Geissel, M. Hegelich, J. Kaae, S. Karsch, G. P. Le Sage, S. Letzring, M. Manclossi, S. Meyroneinc, A. Newkirk, H. Pépin, and N. Renard-LeGalloudec, Phys. Rev. Lett. 92, 204801 (2004).
${ }^{2}$ S. P. Hatchett, C. G. Brown, T. E. Cowan, E. A. Henry, J. S. Johnson, M. H. Key, J. A. Koch, A. Bruce Langdon, B. F. Lasinski, R. W. Lee, A. J. Mackinnon, D. M. Pennington, M. D. Perry, T. W. Phillips, M. Roth, T. Craig Sangster, M. S. Singh, R. A. Snavely, M. A. Stoyer, S. C. Wilks, and K. Yasuike, Phys. Plasmas 7, 2076 (2000).

${ }^{3}$ Th. Sokollik, Investigation of Field Dynamics in Laser Plasmas with Proton Imaging (Springer Verlag, Berlin, 2011).

${ }^{4}$ J. Schreiber, S. Ter-Avetisyan, E. Risse, M. P. Kalachnikov, P. V. Nickles, W. Sandner, U. Schramm, D. Habs, J. Witte, and M. Schnürer, Phys. Plasmas 13, 033111 (2006).

${ }^{5}$ C. Thaury and F. Quéré, J. Phys. B 43, 213001 (2010).

${ }^{6}$ T. Sokollik, M. Schnürer, S. Ter Avetisyan, P. V. Nickles, E. Risse, M. Kalashnikov, W. Sandner, G. Priebe, M. Amin, T. Toncian, O. Willi, and A. A. Andreev, Appl. Phys. Lett. 92, 091503 (2008).

${ }^{7}$ A. P. L. Robinson, P. Foster, D. Adams, D. C. Carroll, B. Dromey, S. Hawkes, S. Kar, Y. T. Li, K. Markey, P. McKenna, C. Spindloe, M. Streeter, C.-G. Wahlström, M. H. Xu, M. Zepf, and D. Neely, New J. Phys. 11, 083018 (2009).

${ }^{8}$ M. P. Kalashnikov, K. Osvay, G. Priebe, L. Ehrentraut, S. Steinke, and W. Sandner, AIP Conf. Proc. 1462, 108 (2012).

${ }^{9}$ S. Ter-Avetisyan, M. Schnürer, and P. V. Nickles, J. Phys. D: Appl. Phys. 38, 863 (2005).

${ }^{10}$ M. Schnürer, S. Ter-Avetisyan, P. V. Nickles, and A. A. Andreev, Phys. Plasmas 14, 033101 (2007).

${ }^{11}$ H. Ruhl, Plasma Sources Sci. Technol. 11, A154 (2002).

${ }^{12}$ P. Mora, Phys. Rev. Lett. 90, 185002 (2003). 